\title{
Políticas de etiquetado en los paquetes de cigarrillos: situación actual en América Latina y el Caribe
}

\author{
Ernesto M Sebrié, MD, MPH. ${ }^{(I)}$
}

Sebrié EM.

Políticas de etiquetado en los paquetes de cigarrillos: situación actual en América Latina y el Caribe. Salud Publica Mex 20 I2;54:293-302.

\section{Resumen}

En 2002, Brasil se convirtió en el primer país de la región en implementar etiquetas de advertencias sanitarias con pictogramas en los paquetes de cigarrillos. Desde la adopción del CMCT de la OMS en 2005, otros nueve países adoptaron advertencias con pictogramas y seis más aprobaron legislación cuya implementación está pendiente. El contenido del mensaje y el estilo del pictograma varían entre los países. Diecisiete países prohibieron descriptores de marca y nueve requieren una advertencia cualitativa con información de constituyentes y emisiones. Un importante progreso se ha alcanzado en la región desde 2005. Sin embargo, los países ratificantes del CMCT aún no han implementado todas las recomendaciones de las Directrices del Artículo II.

Palabras clave: comunicación en salud; legislación sanitaria; políticas públicas; etiquetado de productos derivados del tabaco; envasado de productos derivados del tabaco
Sebrié EM.

Cigarette labeling policies:

current situation in Latin America and the Caribbean.

Salud Publica Mex 2012;54:293-302.

\section{Abstract}

In 2002, Brazil became the first country in the region to implement pictorial health warning labels on cigarette packages. Since the adoption of the FCTC/ WHO in 2005, nine more countries adopted pictorial labels and six passed legislation that is pending of implementation. The message content and the picture style vary across countries. Seventeen countries have banned brand descriptors and nine require a qualitative label with information on constituents and emissions. Since 2005, important progress has been achieved in the region. However, countries that have ratified the FCTC have not yet implemented all the recommendations of Article II Guidelines.

Key words: health communication; health legislation; public policy; tobacco labeling; tobacco packing

(I) Department of Health Behavior, Roswell Park Cancer Institute. Buffalo, New York, EUA.

Fecha de recibido: 6 de marzo de 2012 - Fecha de aceptado: 9 de abril de 2012

Autor de correspondencia: Ernesto M Sebrié, MD MPH. Department of Health Behavior Roswell Park Cancer Institute.

Elm \& Carlton Streets, Buffalo, NY I4263, USA.

Correo electrónico: ernesto.sebrie@roswellpark.org 
E Artículo 11 del Convenio Marco para el Control del Tabaco (CMCT) de la Organización Mundial de la Salud (OMS) adoptado en febrero de 2005 establece las políticas de etiquetado y empaquetado de los productos de tabaco, incluyendo las etiquetas de advertencia sanitaria, la eliminación de información engañosa y las etiquetas de constituyentes y emisiones. ${ }^{1}$ En noviembre de 2008, la tercera Conferencia de las Partes (COP3) aprobó las Directrices para la Implementación del Artículo 11. ${ }^{2}$ Hasta abril de 2012, todos los países de América Latina y el Caribe con excepción de Argentina, Cuba, El Salvador, República Dominicana, Haití y San Vicente \& las Granadinas, habían ratificado el CMCT de la OMS y son Partes del mismo. Tres años después de la ratificación, los países Partes están legalmente obligados a implementar el Artículo 11 de acuerdo con las recomendaciones del CMCT.

Este artículo describe la situación de las políticas de etiquetado en los paquetes de cigarrillos implementadas en los 33 países de América Latina y el Caribe a partir de la legislación vigente hasta abril de 2012, así como el progreso alcanzado a la luz de la adopción del CMCT.

\section{Etiquetas de advertencia sanitaria}

Las etiquetas de advertencia sanitaria difieren en su estilo, número de mensajes y sistema de rotación, localización, tamaño, contenido (incluyendo información gratuita para dejar de fumar), idioma y atribución. El cuadro I muestra un resumen de las características de las etiquetas de advertencia sanitaria implementadas en los 33 países de América Latina y el Caribe hasta abril de 2012.

\section{Estilo}

La mayoría de los países de la región ( $\mathrm{n}=18)$ tiene advertencias de sólo texto impresas en los paquetes de cigarrillos. Siguiendo el ejemplo de Brasil (2002), pionero en la región, nueve países adoptaron una combinación de texto con una fotografía o pictograma: Venezuela (2005), Uruguay (2006), Chile (2006), Panamá (2006), Perú (2009), Colombia (2010), México (2010), Honduras (2011) y Bolivia (2012). Además, Argentina, Ecuador, Nicaragua, El Salvador y Costa Rica han aprobado la legislación, cuya implementación está pendiente, que requiere pictogramas como parte de sus advertencias sanitarias. Los pictogramas implementados en los diez países de la región han adoptado diferentes tipos de imagen para representar el daño, que incluyen desde órganos del cuerpo humano enfermos (por ej. Brasil), imágenes simbólicas o abstractas (por ej. Uruguay), a fotos testimoniales que incluyen el nombre y edad de la persona afectada (por ej. Chile) y de sufrimiento humano donde se ve la cara del fumador o de su familia (por ej. México).

Número de mensajes de advertencia y sistema de rotación

La mayoría de los países ( $\mathrm{n}=18$ ) tienen un único mensaje de advertencia impreso en los paquetes de cigarrillos. El resto tienen más de un mensaje, desde dos en Uruguay a 12 en Jamaica. Uruguay ha adoptado cinco juegos de advertencias con pictogramas múltiples y concurrentes (en 2006, 2007, comienzos de 2009, finales de 2009 y 2012), Brasil y Panamá han adoptado tres juegos (en 2002, 2004, 2009 y en 2006, 2009, 2011, respectivamente) y Venezuela y Colombia dos juegos (en 2005, 2009 y en 2010, 2011, respectivamente). Desde 2006, Chile tiene un par de advertencias dobles (una pictórica en la cara anterior y una sólo texto en la posterior) impresas al mismo tiempo en todos los paquetes de cigarrillos y cada año un nuevo par es introducido (6o. juego en 2011). Desde 2010, México tiene un par de advertencias que sale al mercado cada tres meses y es el sistema de rotación que cambia con mayor frecuencia del mundo.

\section{Localización}

Casi la mitad de los países de la región no tienen regulaciones específicas sobre dónde deben colocarse las advertencias. En estos países las advertencias aparecen generalmente en la cara lateral del paquete o con menor frecuencia en la cara posterior (BAT). El resto de los países tiene diferentes regulaciones, desde ambas caras principales (anterior y posterior) y cara lateral, en el caso de México, hasta sólo una cara principal que generalmente es la posterior como Brasil y Venezuela.

\section{Tamaño}

Al igual que la localización, muchos países no tienen regulaciones específicas sobre el tamaño de las advertencias (que sean de "tamaño legible"). En el resto, el tamaño oscila entre $80 \%$ de ambas caras principales en Uruguay (siendo actualmente las de mayor tamaño en todo el mundo) hasta $25 \%$ de la cara anterior en Guatemala.

\section{Contenido del mensaje}

Casi la mitad de los países $(\mathrm{n}=14)$ requieren una advertencia de sólo texto inespecífica tal como "Fumar es perjudicial para la salud". Un poco más de la mitad $(n=19)$ requieren mensajes con temas relacionados 


\section{Cuadro I}

\section{Características de las etiquetas de advertencia sanitaria en los paquetes de cigarrillos de América Latina y el Caribe (ABRIL de 20I2)}

\begin{tabular}{|c|c|c|c|c|c|c|c|c|}
\hline \multirow{2}{*}{ País } & \multicolumn{8}{|c|}{ Etiquetas de advertencia sanitaria } \\
\hline & Número & Estilo & Rotación & Localización & Tamaño & Idioma & Atribución & Contenido del mensaje \\
\hline $\begin{array}{l}\text { Antigua y } \\
\text { Barbuda }\end{array}$ & I & Sólo texto & No & Lateral & NE & Inglés & $\begin{array}{l}\text { Ministro de } \\
\text { Salud }\end{array}$ & FUMAR ES PELIGROSO PARA LA SALUD \\
\hline Argentina* & 10 & Pictogramas & $\begin{array}{l}\text { Cada } 2 \\
\text { años }\end{array}$ & $\begin{array}{l}\text { Anterior } \\
\text { (inferior) } \\
\text { Posterior } \\
\text { (inferior) }\end{array}$ & $\begin{array}{l}50 \% \\
50 \%\end{array}$ & Español & NE & $\begin{array}{l}\text { I. Fumar causa cáncer } \\
\text { 2. Fumar causa enfisema pulmonar } \\
\text { 3. Fumar causa adicción } \\
\text { 4. Fumar causa impotencia sexual } \\
\text { 5. Fumar causa enfermedades cardiacas y respiratorias } \\
\text { 6. El humo de tabaco es causa de enfermedad y muerte } \\
\text { 7. La mujer embarazada que fuma causa daños irreparables a su hijo } \\
\text { 8. Fumar causa muerte por asfixia } \\
\text { 9. Fumar quita años de vida } \\
\text { 10. Fumar puede causar amputación de piernas }\end{array}$ \\
\hline & I & & & Lateral & & & & Información sobre servicio gratuito para dejar de fumar \\
\hline Bahamas & I & Sólo texto & No & Lateral & NE & Inglés & $\begin{array}{l}\text { Ministro de } \\
\text { Salud }\end{array}$ & FUMAR ES PELIGROSO PARA LA SALUD \\
\hline Barbados & I & Sólo texto & No & Lateral & NE & Inglés & $\begin{array}{l}\text { Ministro de } \\
\text { Salud }\end{array}$ & FUMAR ES PELIGROSO PARA LA SALUD \\
\hline Belice & I & Sólo texto & No & Lateral & $\mathrm{NE}$ & Inglés & $\begin{array}{l}\text { Director de } \\
\text { Servicios de } \\
\text { Salud }\end{array}$ & FUMAR ES PELIGROSO PARA LA SALUD \\
\hline Bolivia & 7 & Pictogramas & Sí & $\begin{array}{l}\text { Anterior } \\
\text { (inferior) } \\
\text { Posterior } \\
\text { (inferior) }\end{array}$ & $\begin{array}{l}50 \% \\
50 \%\end{array}$ & Español & $\begin{array}{l}\text { Ministerio } \\
\text { de Salud y } \\
\text { Deportes }\end{array}$ & $\begin{array}{l}\text { 1. ¡PELIGRO! El humo de tabaco causa asma en los niños. } \\
\text { 2. ¡CUIDADO! Este producto causa envejecimiento precoz de la piel. } \\
\text { 3. ¡CUIDADO! Fumar causa impotencia sexual. } \\
\text { 4. iPELIGRO! Fumar causa cáncer de laringe. } \\
\text { 5. iPELIGRO! Fumando inhalas arsénico y naftalina usado para ratas y } \\
\text { cucarachas. } \\
\text { 6. iPELIGRO! Fumar causa ataque al corazón. } \\
\text { 7. ¡PELIGRO! Fumar causa cáncer de boca. }\end{array}$ \\
\hline Brasil* & 10 & Pictogramas & $\begin{array}{l}\text { Cada } 5 \\
\text { meses }\end{array}$ & Posterior & $100 \%$ & Portugués & $\begin{array}{l}\text { Ministerio de } \\
\text { Salud }\end{array}$ & $\begin{array}{l}\text { I. VICTIMA DE ESTE PRODUCTO - Este producto intoxica a la madre y } \\
\text { al bebé, causando parto prematuro y muerte. } \\
\text { 2. GANGRENA - El uso de este producto obstruye arterias y dificulta la } \\
\text { circulación de la sangre. } \\
\text { 3. MUERTE - El uso de este producto lleva a la muerte por cáncer de } \\
\text { pulmón y enfisema. } \\
\text { 4. INFARTO - El uso de este producto causa muerte por enfermedades } \\
\text { del corazón. } \\
\text { 5. HUMO TÓXICO - Respirar el humo de este producto causa neumonía } \\
\text { y bronquitis. } \\
\text { 6. HORROR - Este producto causa envejecimiento precoz de la piel. } \\
\text { 7. SUFRIMIENTO - La dependencia de la nicotina causa tristeza, dolor y } \\
\text { muerte. } \\
\text { 8. PRODUCTO TÓXICO - Este producto contiene sustancias tóxicas que } \\
\text { llevan a la enfermedad y muerte. } \\
\text { 9. PELIGRO - El riesgo de derrame cerebral es mayor con el uso de este } \\
\text { producto. } \\
\text { I0. IMPOTENCIA - El uso de este producto disminuye, dificulta o impide } \\
\text { la erección. }\end{array}$ \\
\hline & NE & Sólo texto & NE & $\begin{array}{l}\text { Anterior } \\
\text { (inferior) }\end{array}$ & $30 \%$ & Portugués & NE & $\mathrm{NE}$ \\
\hline Colombia & 6 & Pictogramas & Cada año & $\begin{array}{l}\text { Anterior } \\
\text { (inferior) } \\
\text { Posterior } \\
\text { (inferior) }\end{array}$ & $\begin{array}{l}30 \% \\
30 \%\end{array}$ & Español & NE & $\begin{array}{l}\text { 1. Tú escoges el aire que respiras } \\
\text { 2. Fumar causa problemas oculares } \\
\text { 3. Fumar causa impotencia sexual } \\
\text { 4. Tus futuros pulmones } \\
\text { 5. Fumar causa infarto cerebral } \\
\text { 6. Fumar causa aborto }\end{array}$ \\
\hline Costa Rica* & NE & Pictogramas & Sí & $\begin{array}{l}\text { Anterior } \\
\text { Posterior }\end{array}$ & $\begin{array}{l}50 \% \\
50 \%\end{array}$ & Español & NE & NE \\
\hline Cuba & 5 & Sólo texto & Sí & Posterior & $60 \%$ & Español & $\begin{array}{l}\text { Ministerio de } \\
\text { Salud Pública }\end{array}$ & $\begin{array}{l}\text { 1. ¡Detente! Tus hijos están fumando también. } \\
\text { 2. Embarazada, si fumas peligra la vida de tu bebé. } \\
\text { 3. Muestra tu inteligencia, deja de fumar. } \\
\text { 4. Tu aire y el mío es el mismo, ¡respétalo! } \\
\text { 5. ¿Quieres una vida saludable?, ¡No fumes! }\end{array}$ \\
\hline Chile & 2 & Pictogramas & Cada año & $\begin{array}{l}\text { Anterior } \\
\text { Posterior }\end{array}$ & $\begin{array}{l}50 \% \\
50 \%\end{array}$ & Español & $\begin{array}{l}\text { Ministerio } \\
\text { de Salud } \\
\text { Gobierno de } \\
\text { Chile }\end{array}$ & $\begin{array}{l}\text { I. } \quad \text { El tabaco produce cáncer bucal. } \\
\text { 2. } \quad \text { Cuando tú fumas todos mueren. }\end{array}$ \\
\hline
\end{tabular}


(Continuación)

\begin{tabular}{|c|c|c|c|c|c|c|c|c|}
\hline Dominica & 1 & Sólo texto & No & Lateral & NE & Inglés & $\begin{array}{l}\text { Ministro de } \\
\text { Salud }\end{array}$ & FUMAR ES PELIGROSO PARA LA SALUD \\
\hline Ecuador* & 6 & Pictogramas & Cada año & $\begin{array}{l}\text { Anterior } \\
\text { (inferior) } \\
\text { Posterior } \\
\text { (inferior) }\end{array}$ & $60 \%$ & Español & $\begin{array}{l}\text { Ministerio de } \\
\text { Salud Pública }\end{array}$ & $\begin{array}{ll}\text { I. } & \text { Fumar te causa impotencia sexual. } \\
\text { 2. } & \text { Fumar te arruga y daña tu piel. } \\
\text { 3. } & \text { Fumar te causa una muerte lenta y dolorosa. } \\
\text { 4. } & \text { Fumar destruye tu dentadura y tu boca. } \\
\text { 5. } & \text { Fumar intoxica al bebé, causa partos prematuros y muerte. } \\
\text { 6. } & \text { No permitas que el humo de tabaco intoxique a tus hijos. }\end{array}$ \\
\hline El Salvador* & NE & Pictogramas & NE & $\begin{array}{l}\text { Anterior } \\
\text { (inferior) } \\
\text { Posterior } \\
\text { (inferior) }\end{array}$ & $50 \%$ & Español & $\mathrm{NE}$ & NE \\
\hline Granada & 1 & Sólo texto & No & Lateral & $\mathrm{NE}$ & Inglés & $\begin{array}{l}\text { Ministro de } \\
\text { Salud }\end{array}$ & FUMAR ES PELIGROSO PARA LA SALUD \\
\hline Guatemala & 5 & Sólo texto & Sí & $\begin{array}{l}\text { Anterior } \\
\text { (inferior) }\end{array}$ & $25 \%$ & Español & NE & $\begin{array}{l}\text { I. Fumar tabaco produce cáncer. } \\
\text { 2. El consumo de este producto produce enfermedades cardiovasculares. } \\
\text { 3. El consumo de este producto produce cáncer de la boca y de la faringe. } \\
\text { 4. En la mujer embarazada el fumar tabaco produce aborto y } \\
\text { malformación fetal. } \\
\text { 5. El consumo de este producto produce cáncer pulmonar. }\end{array}$ \\
\hline & 1 & Sólo texto & & Lateral & & & & El consumo de este producto causa serios daños a la salud. \\
\hline Guyana & 1 & Sólo texto & No & Lateral & NE & Inglés & $\begin{array}{l}\text { Ministro de } \\
\text { Salud }\end{array}$ & FUMAR ES PELIGROSO PARA LA SALUD \\
\hline Haití & 1 & sólo texto & No & Lateral & $\mathrm{NE}$ & $\begin{array}{l}\text { Francés } \\
\text { y criollo } \\
\text { haitiano }\end{array}$ & NE & FUMAR PERJUDICA GRAVEMENTE A A LA SALUD \\
\hline Honduras & $\mathrm{NE}$ & Pictogramas & Cada año & $\begin{array}{l}\text { Anterior } \\
\text { Posterior }\end{array}$ & $\begin{array}{l}50 \% \\
50 \%\end{array}$ & Español & $\begin{array}{l}\text { Instituto } \\
\text { Hondureño } \\
\text { para la } \\
\text { Prevención } \\
\text { del } \\
\text { Alcoholismo, } \\
\text { Drogadicción } \\
\text { y Farmaco- } \\
\text { dependencia } \\
\text { (IHADFA) }\end{array}$ & $\begin{array}{l}\text { I. CUIDADO "Mensaje de Don Andrés, hondureño, con cáncer de laringe } \\
\text { por fumar". } \\
\text { 2. CUIDADO "Estos cigarrillos te matan". } \\
\text { 3. CUIDADO "Este producto envejece la piel". }\end{array}$ \\
\hline Jamaica & 12 & Sólo texto & Sí & $\begin{array}{l}\text { Anterior } \\
\text { Posterior }\end{array}$ & $\begin{array}{l}30 \% \\
30 \%\end{array}$ & Inglés & $\begin{array}{l}\text { Oficial Jefe } \\
\text { Médico }\end{array}$ & $\begin{array}{l}\text { I. Fumar mata. } \\
\text { 2. Fumar causa cáncer de pulmón. } \\
\text { 3. El humo de tabaco ajeno es dañino para los niños y los no fumadores. } \\
\text { 4. Fumar causa ataques al corazón. } \\
\text { 5. Fumar causa enfermedad pulmonar crónica. } \\
\text { 6. Deje de fumar ahora y mejore su salud. } \\
\text { 7. Fumar causa impotencia. } \\
\text { 8. Fumar es dañino para el bebé por nacer. } \\
\text { 9. Fumar mata a fumadores y otras personas. } \\
\text { 10. Fumar causa ataque cerebral. } \\
\text { II. Fumar causa asma. } \\
\text { I2. Fumar causa cáncer. }\end{array}$ \\
\hline México & $\begin{array}{l}8 \\
8 \\
8\end{array}$ & $\begin{array}{l}\text { Pictogramas } \\
\text { Sólo texto } \\
\text { Sólo texto }\end{array}$ & $\begin{array}{l}\text { Cada } 3 \\
\text { meses }\end{array}$ & $\begin{array}{l}\text { Anterior } \\
\text { Posterior } \\
\text { Lateral }\end{array}$ & $\begin{array}{l}30 \% \\
100 \% \\
100 \%\end{array}$ & Español & NE & $\begin{array}{l}\text { I. CÁNCER DE BOCA. Fumar es la principal causa del cáncer de boca. } \\
\text { Te impide comer, tomar líquidos y te espera una muerte temprana. } \\
\text { 2. INFARTO CARDIACO. Fumar y respirar el humo de tabaco obstruye } \\
\text { las arterias del corazón causando infartos y muerte. } \\
\text { 3. CÁNCER DE GARGANTA. Por fumar se forman tumores en la } \\
\text { garganta. } \\
\text { Muchas veces hay que quitar la laringe, esencial para comer, beber y } \\
\text { hablar... dejas de vivir normalmente. } \\
\text { 4. ENFERMEDADES RESPIRATORIAS } \\
\text { Respirar los tóxicos del humo de tabaco provoca en niños y niñas } \\
\text { enfermedades graves como asma, bronquitis, otitis y neumonía. } \\
\text { 5. ENFISEMA PULMONAR. En México, } 7 \text { de cada I0 personas con } \\
\text { enfisema fumaron. El enfisema destruye tus pulmones, te impide respirar y } \\
\text { realizar tus actividades. } \\
\text { 6. GANGRENA. Al igual que la diabetes, fumar obstruye tus arterias, te } \\
\text { causa gangrena que lleva a la amputación. } \\
\text { 7. CÁNCER DE PULMÓN. En México, el consumo de tabaco es causa de } \\
\text { 9 de cada IO muertes por cáncer de pulmón. } \\
\text { 8. NACIMIENTO PREMATURO. Inhalar humo de tabaco en el embarazo } \\
\text { reduce el oxígeno y nutrientes que llegan al feto. Causa aborto } \\
\text { espontáneo, nacimiento prematuro o daño físico y mental. }\end{array}$ \\
\hline
\end{tabular}




\begin{tabular}{|c|c|c|c|c|c|c|c|c|}
\hline Nicaragua* & 6 & Pictogramas & Cada año & $\begin{array}{l}\text { Anterior } \\
\text { (inferior) } \\
\text { Posterior } \\
\text { (inferior) }\end{array}$ & $\begin{array}{l}50 \% \\
50 \%\end{array}$ & Español & NE & NE \\
\hline & $\mathrm{T}$ & Sólo texto & & Lateral & & & & Fumar es dañino para la salud. \\
\hline Panamá & 5 & Pictogramas & Sí & $\begin{array}{l}\text { Anterior } \\
\text { Posterior }\end{array}$ & $\begin{array}{l}50 \% \\
50 \%\end{array}$ & Español & NE & $\begin{array}{l}\text { I. Fumar puede causar abortos. } \\
\text { 2. Fumar puede causar arrugas prematuras. } \\
\text { 3. Fumar puede causar cáncer de mama. } \\
\text { 4. Fumar puede causar enfermedad cerebrovascular. } \\
\text { 5. Fumar puede causar impotencia. }\end{array}$ \\
\hline Paraguay & $\mathrm{I}$ & Sólo texto & No & Lateral & NE & Español & $\begin{array}{l}\text { Ministerio de } \\
\text { Salud Pública } \\
\text { y Bienestar } \\
\text { Social }\end{array}$ & Fumar daña la salud \\
\hline Perú & 12 & Pictogramas & Cada año & $\begin{array}{l}\begin{array}{l}\text { Anterior } \\
\text { (superior) }\end{array} \\
\text { Posterior } \\
\text { (superior) }\end{array}$ & $\begin{array}{l}50 \% \\
50 \%\end{array}$ & Español & NE & $\begin{array}{l}\text { I. Fumar causa gangrena. } \\
\text { 2. Fumar causa cáncer de mama. } \\
\text { 3. Fumar causa impotencia sexual. } \\
\text { 4. Fumar causa aborto. } \\
\text { 5. Fumar causa cáncer de pulmón. } \\
\text { 6. El humo del tabaco causa asma en los niños. } \\
\text { 7. El humo de tabaco daña tu bebé. } \\
\text { 8. La nicotina es más adictiva que la heroína. } \\
\text { 9. Fumar causa infarto al corazón. } \\
\text { I0. Fumar causa cáncer de laringe. } \\
\text { II. Fumar causa infarto cerebral. } \\
\text { I2. Fumar causa ceguera. }\end{array}$ \\
\hline $\begin{array}{l}\text { República } \\
\text { Dominicana }\end{array}$ & I & Sólo texto & No & Lateral & NE & Español & $\mathrm{NE}$ & Fumar es perjudicial para la salud \\
\hline $\begin{array}{l}\text { San } \\
\text { Cristóbal y } \\
\text { Nieves }\end{array}$ & I & Sólo texto & No & Lateral & NE & Inglés & $\begin{array}{l}\text { Ministro de } \\
\text { Salud }\end{array}$ & FUMAR ES PELIGROSO PARA LA SALUD \\
\hline Santa Lucía & $\mathrm{I}$ & Sólo texto & No & Lateral & NE & Inglés & $\begin{array}{l}\text { Ministro de } \\
\text { Salud }\end{array}$ & FUMAR ES PELIGROSO PARA LA SALUD \\
\hline $\begin{array}{l}\text { San Vicente } \\
\text { y las } \\
\text { Granadinas }\end{array}$ & $\mathrm{I}$ & Sólo texto & No & Lateral & $\mathrm{NE}$ & Inglés & $\begin{array}{l}\text { Ministro de } \\
\text { Salud }\end{array}$ & FUMAR ES PELIGROSO PARA LA SALUD \\
\hline Surinam & I & Sólo texto & No & Lateral & $\mathrm{NE}$ & Holandés & $\begin{array}{l}\text { Ministro de } \\
\text { Salud }\end{array}$ & $\begin{array}{l}\text { ADVERTENCIA: EL CONSUMO DE TABACO PUEDE CAUSAR } \\
\text { GRAVES DAÑOS A LA SALUD }\end{array}$ \\
\hline $\begin{array}{l}\text { Trinidad y } \\
\text { Tobago* }\end{array}$ & $\mathrm{NE}$ & $\mathrm{NE}$ & No & NE & NE & Inglés & NE & $\mathrm{NE}$ \\
\hline Uruguay & 2 & Pictogramas & Sí & $\begin{array}{l}\text { Anterior } \\
\text { (inferior) } \\
\text { Posterior } \\
\text { (inferior) } \\
\text { Anterior }\end{array}$ & $\begin{array}{l}80 \% \\
80 \%\end{array}$ & Español & $\begin{array}{l}\text { Ministerio de } \\
\text { Salud Pública }\end{array}$ & $\begin{array}{l}\text { I. Fumar causa infartos cerebrales. } \\
\text { 2. El humo de tabaco enferma a tus hijos. Los niños expuestos al humo de } \\
\text { tabaco tienen un riesgo aumentado de sufrir asma, neumonía, bronquitis e } \\
\text { infecciones de oído. }\end{array}$ \\
\hline Venezuela & 10 & $\begin{array}{l}\text { Logo y texto } \\
\text { Pictogramas }\end{array}$ & Sí & $\begin{array}{l}\text { Anterior } \\
\text { Posterior }\end{array}$ & $100 \%$ & Español & $\begin{array}{l}\text { Ministerio } \\
\text { del Poder } \\
\text { Popular para } \\
\text { la Salud y } \\
\text { Protección } \\
\text { Social }\end{array}$ & $\begin{array}{l}\text { Producto tóxico } \\
\text { I. Los niños y niñas comienzan a fumar al ver adultos fumando. } \\
\text { 2. Este producto es dañino para la salud y produce adicción. } \\
\text { 3. Fumar durante el embarazo daña la salud de tu bebé. } \\
\text { 4. El humo del cigarrillo afecta también a quien no fuma. } \\
\text { 5. Fumar causa infarto al corazón. } \\
\text { 6. Fumar causa cáncer de pulmón, tos, enfisema pulmonar y bronquitis } \\
\text { crónica. } \\
\text { 7. Fumar causa mal aliento, pérdida de muelas y cáncer de boca. } \\
\text { 8. Fumar cigarrillos causa cáncer de laringe. } \\
\text { 9. Fumar cigarrillos causa impotencia en los hombres. } \\
\text { 10. Da hoy el primer paso, dejar de fumar es posible. }\end{array}$ \\
\hline
\end{tabular}

* Regulación o implementación (total o parcial) pendiente

NE: no especificado

Fuente: referencias 3 y 4

a efectos sobre la salud, incluyendo mensajes sobre cesación tabáquica y sobre las consecuencias de la exposición pasiva al humo de tabaco ajeno. A partir de 2009, los paquetes de Venezuela también incluyen un logo con el mensaje "Venezuela Libre de Humo de Tabaco" y a partir de 2010, los paquetes de Uruguay tienen un pictograma de una calavera con dos huesos cruzados con la leyenda "Producto Tóxico" (símbolo reconocido internacionalmente para las sustancias venenosas). Ningún país requiere mensajes relacionados al impacto medioambiental, económico o sobre las estrategias de la industria tabacalera tal como es recomendado en las Directrices del Artículo 11 del CMCT. 
Información gratuita para dejar de fumar

Un número telefónico gratuito para dejar de fumar es requerido en los paquetes de Brasil, Uruguay, México y Argentina. Además, los paquetes en Uruguay incluyen una página de internet donde los fumadores pueden obtener información sobre cesación tabáquica.

Idioma

La mayoría de los países de la región tienen advertencias escritas en una sola lengua oficial, ya sea español para los países latinoamericanos, portugués en Brasil, inglés en la mayoría de los países del Caribe y holandés en Surinam. Sólo Haití tiene advertencias en dos idiomas oficiales: francés y criollo haitiano.

\section{Atribución}

A pesar de no ser un requerimiento del Artículo 11, más de la mitad $(\mathrm{n}=20)$ de los países atribuyen sus advertencias a una autoridad de salud nacional o una provisión legal. Agencias de salud incluyen el Ministerio de Salud (por ej. en Barbados), el Instituto Hondureño para la Prevención del Alcoholismo Drogadicción y Farmacodependencia (IHADFA) (por ej. en Honduras), el Ministerio de Salud Pública y Bienestar Social (por ej. en El Salvador), y el Oficial Jefe Médico (Chief Medical Officer) (por ej. en Jamaica).

\section{Prohibición de información engañosa}

Las compañías tabacaleras usualmente imprimen mensajes, símbolos, colores, números u otra información o aspectos del diseño en los paquetes de cigarrillos que pueden engañar a los consumidores e inducirlos a creer que hay productos de tabaco que tienen niveles de sustancias de menor riesgo para la salud o incluso que existe un tiempo seguro para el consumo del tabaco. El cuadro II muestra un resumen de las prohibiciones de información engañosa en el empaquetado de cigarrillos implementadas en los 33 países de América Latina y el Caribe hasta abril de 2012.

\section{Descriptores de marca}

Siguiendo las Directrices del Artículo 11 del CMCT, casi la mitad de los países ( $\mathrm{n}=17)$ han prohibido los descriptores de marca con referencias implícitas a reducción de daño tales como "light", "mild", suaves, ultrasuaves, bajo contenido en alquitrán, etc. Además, Bolivia ha sido el único país en la región en prohibir afirmaciones de "libre de aditivos", 100\% natural, y tabaco orgánico.
Brasil ha prohibido el uso de números como descriptores de marca ya que pueden dar la falsa impresión de ser productos menos riesgosos para la salud.

\section{Familias de marcas y colores}

Uruguay ha sido el único país que prohíbe el uso de colores para identificar diferentes tipos de cigarrillos dentro de una familia de marca permitiendo sólo una única presentación por familia de marca. La transnacional tabacalera Philip Morris International está litigando contra el gobierno de Uruguay por esta medida que es única en el mundo y que sigue la lógica del empaquetado genérico.

Entregas cuantitativas de alquitrán, nicotina y monóxido de carbono

Sólo Bolivia, Panamá y Ecuador han prohibido el uso de entregas cuantitativas de alquitrán, nicotina, monóxido de carbono u otras emisiones, siguiendo la misma lógica que Brasil en la cual los valores más bajos pueden dar la falsa impresión de ser productos menos riesgosos para la salud.

\section{Fecha de vencimiento}

Colombia es el único país de la región que ha prohibido la colocación de una fecha de vencimiento para el consumo del producto, la cual puede llevar engañosamente a los consumidores a creer que hay un tiempo seguro para consumir tabaco.

\section{Etiquetas de constituyentes tóxicos $y$ emisiones}

La información sobre constituyentes tóxicos y emisiones es requerida por ley o impresa voluntariamente por las compañías tabacaleras en los paquetes de cigarrillos. Se presentan dos tipos de información: entregas cuantitativas e información cualitativa. El cuadro III muestra un resumen de las características de las etiquetas de constituyentes y emisiones en el empaquetado de cigarrillos implementadas en los 33 países de América Latina y el Caribe hasta abril de 2012.

\section{Entregas cuantitativas (cantidades numéricas)}

Información cuantitativa de las entregas de alquitrán, nicotina y monóxido de carbono es impresa voluntariamente por la industria del tabaco en al menos 11 países. A pesar de que las Directrices del Artículo 11 del CMCT recomiendan su prohibición, seis países lo requieren por ley. 
Cuadro II

Prohibición de información engañosa en los paQuetes de cigarrillos de América Latina y el Caribe (ABRIL de 20I2)

\begin{tabular}{|c|c|c|c|}
\hline \multirow[b]{2}{*}{ País } & \multicolumn{3}{|c|}{ Información engañosa } \\
\hline & $\begin{array}{l}\text { Descriptores de marca con referencia a } \\
\text { reducción de daño }\end{array}$ & Entregas de componentes y emisiones & Fecha de vencimiento para el consumo \\
\hline Antigua y Barbuda & - & - & - \\
\hline Argentina* & Prohibido & - & - \\
\hline Bahamas & - & - & - \\
\hline Barbados & - & - & - \\
\hline Belice & - & - & - \\
\hline Bolivia & $\begin{array}{l}\text { Prohibido incluyendo "Sin aditivos", } \\
\text { "I00\% natural" y "orgánico" }\end{array}$ & Prohibido & - \\
\hline Brasil & $\begin{array}{c}\text { Prohibido incluyendo el uso de } \\
\text { números }\end{array}$ & - & - \\
\hline Chile & Prohibido & - & - \\
\hline Colombia & Prohibido & - & Prohibido \\
\hline Costa Rica* & Prohibido & - & - \\
\hline Cuba & Prohibido & - & - \\
\hline Dominica & - & - & - \\
\hline Ecuador* & Prohibido & Prohibido & - \\
\hline El Salvador* & Prohibido & - & - \\
\hline Granada & - & - & - \\
\hline Guatemala & - & - & - \\
\hline Guyana & - & - & - \\
\hline Haití & - & - & - \\
\hline Honduras & Prohibido & - & - \\
\hline Jamaica & - & - & - \\
\hline México & Prohibido & - & - \\
\hline Nicaragua* & Prohibido & - & - \\
\hline Panamá & Prohibido & Prohibido & - \\
\hline Paraguay & - & - & - \\
\hline Perú & Prohibido & - & - \\
\hline República Dominicana & - & - & - \\
\hline San Cristóbal y Nieves & - & - & - \\
\hline Santa Lucía & - & - & - \\
\hline San Vicente y las Granadinas & - & - & - \\
\hline Surinam & - & - & - \\
\hline Trinidad y Tobago* & Prohibido & - & - \\
\hline Uruguay & $\begin{array}{c}\text { Prohibido incluyendo el uso de } \\
\text { colores }\end{array}$ & - & - \\
\hline Venezuela & Prohibido & - & - \\
\hline
\end{tabular}

\section{Etiquetas cualitativas o descriptivas}

Once países requieren una leyenda impresa en la cara lateral o en la cara posterior, con información cualitativa sobre los constituyentes tóxicos como alquitrán, nicotina y monóxido de carbono siguiendo las recomendaciones de las Directrices del Artículo 11 del CMCT. Además, Chile, Bolivia, Uruguay y México también revelan información sobre otros carcinógenos diferentes del alquitrán tales como arsénico, cadmio y polonio. México y Perú también proveen información sobre otras sustancias tóxicas tales como cianuro. México provee información en su cara posterior de tóxicos como amoníaco, talio, polonio 210, tolueno, benzopireno, butano, cromo y formol. Ecuador y Costa Rica aún no han reglamentado qué información cualitativa proveerán en sus paquetes de cigarrillos. 


\section{Cuadro III \\ Características de las etiquetas de constituyentes y emisiones en los paquetes de cigarrillos de América Latina y el Caribe (abril de 20I2)}

\begin{tabular}{|c|c|c|c|c|}
\hline \multirow[b]{2}{*}{ País } & \multicolumn{4}{|c|}{ Información de constituyentes y emisiones } \\
\hline & Estilo & Localización & Tamaño & Contenido \\
\hline Antigua y Barbuda & Entregas cuantitativas & Lateral & $\mathrm{NE}$ & Alquitrán y nicotina \\
\hline Argentina & Entregas cuantitativas & Lateral & NE & Alquitrán, nicotina, monóxido de carbono \\
\hline Bahamas & Entregas cuantitativas & Lateral & NE & Alquitrán y nicotina \\
\hline Barbados & Entregas cuantitativas & Lateral & NE & Alquitrán y nicotina \\
\hline Belice & Entregas cuantitativas & Lateral & NE & Alquitrán y nicotina \\
\hline Bolivia & Descriptivo & Lateral & $50 \%$ & Alquitrán, nicotina, monóxido de carbono y arsénico \\
\hline Brasil & Descriptivo & Lateral & NE & 4700 sustancias tóxicas y nicotina \\
\hline Chile & Descriptivo & Lateral & $100 \%$ & Alquitrán, nicotina, monóxido de carbono y arsénico \\
\hline Colombia & NE & NE & NE & NE \\
\hline Costa Rica* & Descriptivo & Lateral & $100 \%$ & NE \\
\hline Cuba & Entregas cuantitativas & NE & NE & Alquitrán, nicotina y monóxido de carbono \\
\hline Dominica & Entregas cuantitativas & Lateral & NE & Alquitrán y nicotina \\
\hline Ecuador* & Descriptivo & Lateral & $100 \%$ & $\begin{array}{l}\text { 70\%: } 4000 \text { tóxicos, arsénico, monóxido de carbono, } \\
\text { cianuro, amoniaco, } 50 \text { sustancias cancerígenas. } \\
\text { 30\%: icono de calavera y huesos, producto tóxico }\end{array}$ \\
\hline El Salvador* & Entregas cuantitativas & Lateral & $\begin{array}{l}\text { No menor de } \\
10 \mathrm{~mm}\end{array}$ & Nicotina, alquitrán y monóxido de carbono \\
\hline Granada & Entregas cuantitativas & Lateral & NE & Alquitrán y nicotina \\
\hline Guatemala & NE & NE & $\mathrm{NE}$ & NE \\
\hline Guyana & Entregas cuantitativas & Lateral & NE & Alquitrán y nicotina \\
\hline Haití & $\mathrm{NE}$ & $\mathrm{NE}$ & $\mathrm{NE}$ & $\mathrm{NE}$ \\
\hline Honduras & Descriptivo & Lateral & NE & Alquitrán, nicotina y monóxido de carbono \\
\hline Jamaica & Entregas cuantitativas & Lateral & NE & Alquitrán, nicotina y monóxido de carbono \\
\hline México & Descriptivo & Posterior & $100 \%$ & $\begin{array}{l}\text { Talio, alquitrán, } 4000 \text { sustancias venenosas y } \\
\text { cancerígenas, amoniaco, cadmio, cianuro, polonio } 210 \text {, } \\
\text { tolueno, benzopireno, butano, cromo y formol }\end{array}$ \\
\hline Nicaragua* & Entregas cuantitativas & Lateral & NE & Alquitrán, nicotina y monóxido de carbono \\
\hline Panamá & Descriptivo & Lateral & $100 \%$ & $\begin{array}{l}\text { Alquitrán, nicotina, monóxido de carbono y } \\
\text { benzopireno }\end{array}$ \\
\hline Paraguay & Entregas cuantitativas & Lateral & NE & Alquitrán, nicotina, monóxido de carbono \\
\hline Perú & Descriptivo & Lateral & $100 \%$ & $\begin{array}{l}\text { Más de } 4000 \text { sustancias tóxicas, } 50 \text { cancerígenas, } \\
\text { alquitrán, cianuro, nicotina y monóxido de carbono }\end{array}$ \\
\hline República Dominicana & NE & NE & NE & NE \\
\hline San Cristóbal y Nieves & Entregas cuantitativas & Lateral & NE & Alquitrán y nicotina \\
\hline Santa Lucía & Entregas cuantitativas & Lateral & NE & Alquitrán y nicotina \\
\hline San Vicente y las Granadinas & Entregas cuantitativas & Lateral & NE & Alquitrán y nicotina \\
\hline Surinam & Entregas cuantitativas & Lateral & NE & Alquitrán y nicotina \\
\hline Trinidad y Tobago* & NE & NE & NE & Constituyentes y aditivos \\
\hline Uruguay & Descriptivo & Lateral & $100 \%$ & Alquitrán, nicotina y monóxido de carbono \\
\hline Venezuela & Descriptivo & Lateral & $100 \%$ & Alquitrán, nicotina y monóxido de carbono \\
\hline $\begin{array}{l}\text { * Implementación pendiente } \\
\text { NE: no especificado } \\
\text { Fuente: referencias } 3 \text { y } 4\end{array}$ & & & & \\
\hline
\end{tabular}




\section{Conclusiones}

En 2002, Brasil se convirtió en el primer país de América Latina y el Caribe en implementar etiquetas de advertencias sanitarias con pictogramas en los paquetes de cigarrillos. A partir de la adopción del CMCT en 2005, otros nueve países adoptaron advertencias con pictogramas y cinco más aprobaron legislación en esa dirección (aunque su implementación estaba pendiente al momento de elaborar este artículo), totalizando casi la mitad de los países de la región (15/33). El contenido de los mensajes, estilo del pictograma, tamaño, localización, número y sistema de rotación varían entre los países. Alrededor de la mitad han adoptado advertencias sanitarias con un sistema de rotación periódico (17/33), tienen un tamaño de al menos $50 \%$ de la superficie total del paquete (14/33) y están localizadas en las dos caras principales del mismo (15/33) (figura 1). Poco más de la mitad de los países (17/33) prohibieron descriptores de marca tales como "light", "suaves" o "bajo en alquitrán", pero solamente Bolivia, Panamá y Ecuador han prohibido la impresión de entregas cuantitativas de alquitrán, nicotina, monóxido de carbono u otras emisiones, y Colombia lo hizo con la fecha de vencimiento para el consumo del producto (figura 2). Un tercio de los países $(11 / 33)$ requieren una advertencia lateral (excepto en México que es posterior) con información cualitativa sobre constituyentes y emisiones del tabaco (figura 3).

Desde la adopción del CMCT de la OMS se ha logrado un importante progreso en la región de América Latina y el Caribe en relación con la implementación efectiva del Artículo 11, especialmente si se la compara con otras regiones del mundo. Sin embargo, ninguno de los países ratificantes del CMCT (27/33) ha implementado aún todas las recomendaciones de las Directrices del Artículo 11. De los 20 países de la subregión latinoamericana, Bolivia, Brasil, Chile, Honduras, México, Panamá, Perú, Uruguay y Venezuela han sido los que más han avanzado al pretender implementar todo el paquete de políticas de etiquetado de productos del tabaco recomendado por el CMCT, mientras que el resto adoptaron solamente algunas de las medidas. De los 13 países de la subregión del Caribe de habla inglesa, Jamaica es el único que ha avanzado en la adopción de políticas de etiquetado de acuerdo con el CMCT, y Trinidad y Tobago, aunque aprobó nueva legislación en 2009, aún no la ha reglamentado ni implementado. Finalmente, es interesante destacar que Argentina y El Salvador, dos de los países que aún no son Partes del $\mathrm{CMCT}$, han aprobado nuevas políticas de etiquetado aunque esté pendiente su implementación.

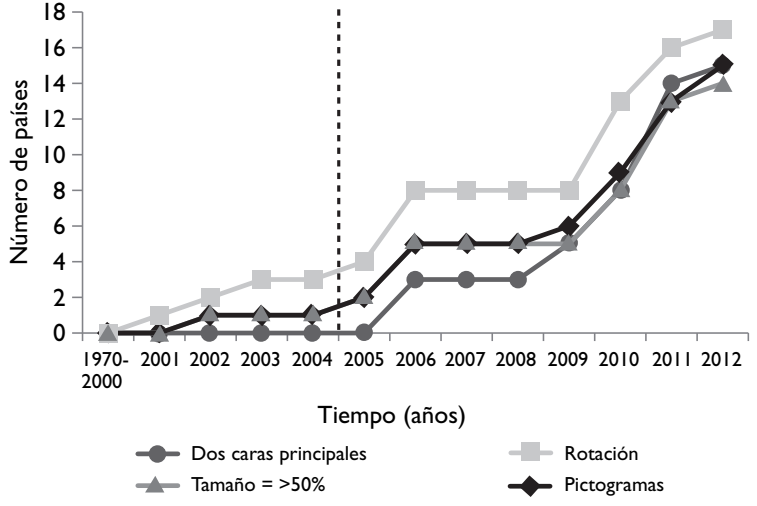

Nota: La línea vertical indica la adopción del CMCT (feb 2005)

Figura I. ETIQUetas de ADVERTENCIAS SANITARIAS

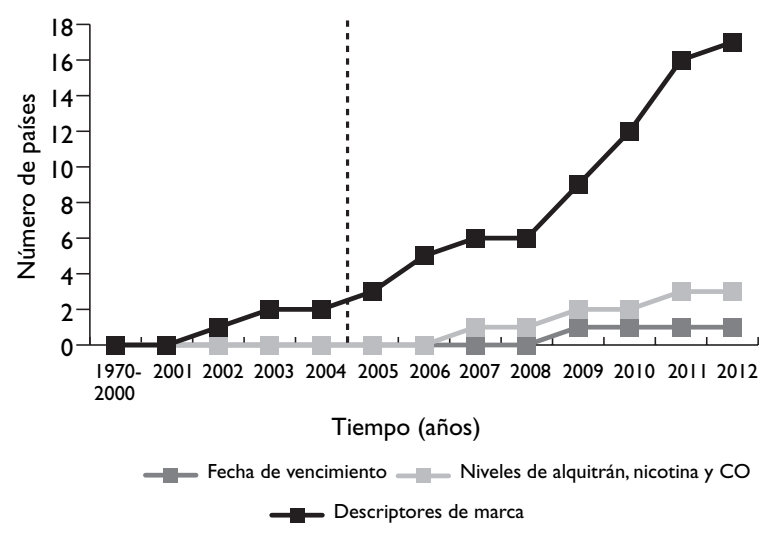

Nota: La línea vertical indica la adopción del CMCT (feb 2005)

Figura 2. Prohibición de información engañosa

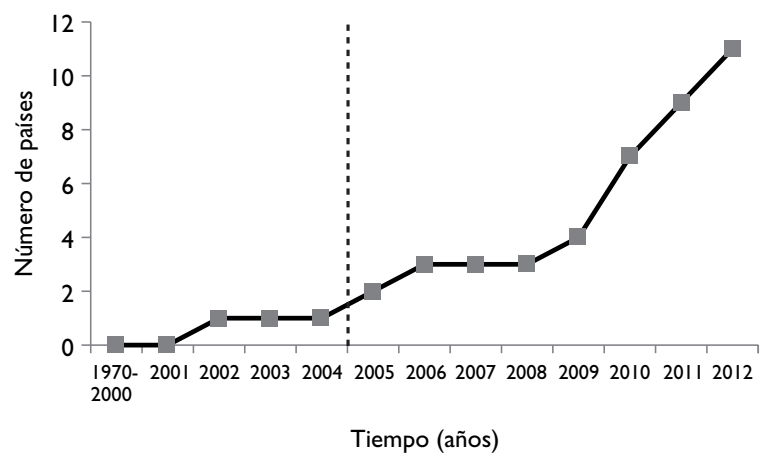

Nota: La línea vertical indica la adopción del CMCT (feb 2005)

Figura 3. Etiquetas de información descriptiva de CONSTITUYENTES Y EMISIONES 


\section{Agradecimientos}

Esta investigación fue financiada por el Flight Attendant Medical Research Institute (FAMRI) y un grant del Instituto Nacional del Cáncer P01 CA138389 "Effectiveness of Tobacco Control Policies in High vs. Low Income Countries". Las agencias financiadoras no tuvieron injerencia en el desarrollo de esta investigación ni en la preparación del manuscrito.

Declaración de conflicto de intereses: El autor declaró no tener conflicto de intereses.

\section{Referencias}

I. World Health Organization. Framework Convention on Tobacco Control. Geneva: WHO; 2003. [Accesado 2010, Marzo 20] Disponible en: http://www.who.int/tobacco/framework/download/en/index.html 2. World Health Organization. Guidelines for implementation of Article II of the WHO Framework Convention on Tobacco Control (packaging and labelling of tobacco products). Durban; 2008 Nov 17-22. [Accesado 2010, Marzo 20] Disponible en: http://www.who.int/fctc/guidelines/ article_I l/en/index.html

3. Sebrié E, Blanco A, Glantz S. Cigarette labeling policies in Latin America and the Caribbean: progress and obstacles. Salud Publica Mex 2010;52 (Sup 2): 233-243.

4. International Legal Consortium, Campaign Tobacco-Free Kids, Washington DC [Accesado 2012, Mayo]. Disponible en: http://www. tobaccocontrollaws.org 